\title{
ZETA FUNCTIONS AND NORMALIZED MULTIPLE SINE FUNCTIONS
}

\author{
Shin-ya Koyama and Nobushige Kurokawa
}

\begin{abstract}
By using normalized multiple sine functions we show expressions for special values of zeta functions and $L$-functions containing $\zeta(3), \zeta(5)$, etc. Our result reveals the importance of division values of normalized multiple sine functions. Properties of multiple Hurwitz zeta functions are crucial for the proof.
\end{abstract}

\section{Introduction}

The normalized sine function

$$
S_{1}(x)=2 \sin (\pi x)
$$

has the basic importance in number theory. This is expressed as

$$
S_{1}(x)=\Gamma_{1}(x)^{-1} \Gamma_{1}(1-x)^{-1}
$$

with the normalized gamma function

$$
\Gamma_{1}(x)=\exp \left(\left.\frac{\partial}{\partial s} \zeta(s, x)\right|_{s=0}\right),
$$

where

$$
\zeta(s, x)=\sum_{n=0}^{\infty}(n+x)^{-s}
$$

is the Hurwitz zeta function. In fact, Lerch's formula says that

$$
\Gamma_{1}(x)=\frac{\Gamma(x)}{\sqrt{2 \pi}}
$$

for the usual gamma function $\Gamma(x)$.

We know that the special value of $S_{1}(x)$ at a rational number $x \in \mathbf{Q}$ with $0<x<1$ is an algebraic integer

Received October 25, 2004; revised March 22, 2005. 


$$
\begin{aligned}
S_{1}(x) & =\left(e^{-\pi i x}-e^{\pi i x}\right) i \\
& =\left|1-e^{2 \pi i x}\right| .
\end{aligned}
$$

(Since $S_{1}(1 / 3)=\sqrt{3}$, the factor " 2 " is needed in $S_{1}(x)$ to assure the integrality.) This algebraic integer is intimately related to the cyclotomic unit, and at the same time it appears in the socalled the class number formula of Dirichlet

$$
L(1, \chi)=-\frac{\tau(\chi)}{N} \log \left(\prod_{k=1}^{N-1} S_{1}\left(\frac{k}{N}\right)^{\bar{\chi}(k)}\right)
$$

where

$$
L(s, \chi)=\sum_{n=1}^{\infty} \chi(n) n^{-s}
$$

is the Dirichlet $L$-function for a non-trivial primitive even Dirichlet character $\chi$ modulo $N$ and

$$
\tau(\chi)=\sum_{k=1}^{N-1} \chi(k) e^{2 \pi i k / N}
$$

is the Gauss sum. We notice that the Dirichlet's formula is written also as

$$
L^{\prime}(0, \chi)=-\frac{1}{2} \log \left(\prod_{k=1}^{N-1} S_{1}\left(\frac{k}{N}\right)^{\chi(k)}\right)
$$

via the functional equation.

The purpose of this paper is to generalize such a formula to the case of $L(r, \chi)$ for $r \geq 2$ containing the Riemann zeta case $\chi=1$ by using the normalized multiple sine function $S_{r}(x)$, which was constructed and studied in the previous paper [KK] (see $\$ 2$ for a survey). We recall the construction. For $\omega_{1}, \ldots, \omega_{r}>0$ and $x>0$, the multiple Hurwitz zeta function is defined by Barnes [B] as

$$
\zeta_{r}\left(s, x ;\left(\omega_{1}, \ldots, \omega_{r}\right)\right)=\sum_{n_{1}, \ldots, n_{r}=0}^{\infty}\left(n_{1} \omega_{1}+\cdots+n_{r} \omega_{r}+x\right)^{-s}
$$

in $\operatorname{Re}(s)>r$. This has the analytic continuation to all $s \in \mathbf{C}$ as a meromorphic function, and it is holomorphic at $s=0$. Then the normalized multiple gamma function is defined as

$$
\Gamma_{r}\left(x,\left(\omega_{1}, \ldots, \omega_{r}\right)\right)=\exp \left(\left.\frac{\partial}{\partial s} \zeta_{r}\left(s, x ;\left(\omega_{1}, \ldots, \omega_{r}\right)\right)\right|_{s=0}\right) .
$$

This is a constant multiple of multiple gamma function $\Gamma_{r}^{B}\left(x ;\left(\omega_{1}, \ldots, \omega_{r}\right)\right)$ of Barnes [B]: 


$$
\Gamma_{r}\left(x ;\left(\omega_{1}, \ldots, \omega_{r}\right)\right)=\Gamma_{r}^{B}\left(x ;\left(\omega_{1}, \ldots, \omega_{r}\right)\right) / \rho_{r}\left(\omega_{1}, \ldots, \omega_{r}\right) .
$$

Now, the normalized multiple sine function is

$$
S_{r}\left(x ;\left(\omega_{1}, \ldots, \omega_{r}\right)\right)=\Gamma_{r}\left(x ;\left(\omega_{1}, \ldots, \omega_{r}\right)\right)^{-1} \Gamma_{r}\left(\omega_{1}+\cdots+\omega_{r}-x ;\left(\omega_{1}, \ldots, \omega_{r}\right)\right)^{(-1)^{r}} .
$$

Hence, by the zeta regularized product (see Manin $[\mathbf{M}]$ ), we can write

$$
\begin{aligned}
S_{r}\left(x ;\left(\omega_{1}, \ldots, \omega_{r}\right)\right) & \\
\quad= & \prod_{n_{1}, \ldots, n_{r}=0}^{\infty}\left(n_{1} \omega_{1}+\cdots+n_{r} \omega_{r}+x\right)\left(\prod_{n_{1}, \ldots, n_{r}=1}^{\infty}\left(n_{1} \omega_{1}+\cdots+n_{r} \omega_{r}-x\right)\right)^{(-1)^{r-1}} .
\end{aligned}
$$

For example

$$
\begin{aligned}
S_{1}(x, \omega) & =\Gamma_{1}(x, \omega)^{-1} \Gamma_{1}(\omega-x, \omega)^{-1} \\
& =\prod_{n=0}^{\infty}(n \omega+x) \prod_{n=1}^{\infty}(n \omega-x) \\
& =2 \sin (\pi x / \omega)
\end{aligned}
$$

since we have

$$
\Gamma_{1}(x, \omega)=(2 \pi)^{-1 / 2} \Gamma(x / \omega) \omega^{x / \omega-1 / 2}
$$

from

$$
\zeta_{1}(s, x, \omega)=\omega^{-s \zeta}(s, x / \omega) .
$$

To simplify the notation we put $S_{r}(x)=S_{r}(x ;(1, \ldots, 1)), \Gamma_{r}(x)=\Gamma_{r}(x ;(1, \ldots, 1))$ and $\zeta_{r}(s, x)=\zeta_{r}(s, x ;(1, \ldots, 1))$. Hence

$$
S_{r}(x)=\Gamma_{r}(x)^{-1} \Gamma_{r}(r-x)^{(-1)^{r}}
$$

and

$$
\Gamma_{r}(x)=\exp \left(\left.\frac{\partial}{\partial s} \zeta_{r}(s, x)\right|_{s=0}\right) .
$$

This normalized multiple sine function $S_{r}(x)$ has good properties similar to the usual sine function $S_{1}(x)=2 \sin (\pi x)$. We refer to $\$ 2$ for details. For example, it has the periodicity and the duplication formula:

$$
S_{r}(x+1)=S_{r}(x) S_{r-1}(x)^{-1}
$$

and

$$
S_{r}(2 x)=\prod_{k=0}^{r} S_{r}\left(x+\frac{k}{2}\right)^{\left(\begin{array}{l}
r \\
k
\end{array}\right)} .
$$

Moreover $S_{r}(x)$ satisfies the following differential equation: 


$$
\frac{S_{r}^{\prime}(x)}{S_{r}(x)}=Q_{r}(x) \cot \pi x
$$

with $Q_{r}(x)=(-1)^{r-1} \pi\left(\begin{array}{l}x-1 \\ r-1\end{array}\right)$. So, $S_{r}(x)$ is a solution of the algebraic differential equation

$$
S_{r}^{\prime \prime}(x)+\left(\pi Q_{r}(x)^{-1}-1\right) S_{r}^{\prime}(x)^{2} S_{r}(x)^{-1}-Q_{r}^{\prime}(x) Q_{r}(x)^{-1} S_{r}^{\prime}(x)+\pi Q_{r}(x) S_{r}(x)=0 .
$$

We also note that $S_{r}(x)$ has the Weierstrass product expression similar to

$$
\begin{aligned}
S_{1}(x) & =2 \pi x \prod_{n=1}^{\infty}\left(1-\frac{x^{2}}{n^{2}}\right) \\
& =2 \pi x \prod_{n=1}^{\infty}\left(\left(1+\frac{x}{n}\right)^{{ }_{1} H_{n}}\left(1-\frac{x}{n}\right)^{1^{1} H_{-n}}\right) .
\end{aligned}
$$

For example

$$
\begin{aligned}
S_{2}(x) & =2 \pi x e^{-x} \prod_{n=1}^{\infty}\left(\left(1+\frac{x}{n}\right)^{n+1}\left(1-\frac{x}{n}\right)^{-n+1} e^{-2 x}\right) \\
& =2 \pi x e^{-x} \prod_{n=1}^{\infty}\left(\left(1+\frac{x}{n}\right)^{2 H_{n}}\left(1-\frac{x}{n}\right)^{2 H_{-n}} e^{-2 x}\right)
\end{aligned}
$$

and

$$
\begin{aligned}
S_{3}(x) & =2 \pi e^{-\zeta^{\prime}(-2)} x e^{x^{2} / 4-(3 / 2) x} \prod_{n=1}^{\infty}\left(\left(1+\frac{x}{n}\right)^{n^{2} / 2+3 n / 2+1}\left(1-\frac{x}{n}\right)^{n^{2} / 2-3 n / 2+1} e^{x^{2} / 2-3 x}\right) \\
& =2 \pi e^{-\zeta^{\prime}(-2)} x e^{x^{2} / 4-(3 / 2) x} \prod_{n=1}^{\infty}\left(\left(1+\frac{x}{n}\right)^{3 H_{n}}\left(1-\frac{x}{n}\right)^{3 H_{-n}} e^{x^{2} / 2-3 x}\right)
\end{aligned}
$$

(see $\S 2)$.

Our main results are as follows. The first result expresses the values of the Riemann zeta function at positive odd integers.

THEOREM 1.1. Let $n=1,2,3, \ldots$, and for $k=1,2, \ldots, n$ put

$$
a(2 n+1, k)=\sum_{l=1}^{k}(-1)^{k-l} l^{2 n}\left(\begin{array}{c}
2 n+1 \\
k-l
\end{array}\right),
$$

which is a positive integer. Then we have:

(1)

$$
\zeta^{\prime}(-2 n)=-\log \left(\prod_{k=1}^{n} S_{2 n+1}(k)^{a(2 n+1, k)}\right)
$$


(2)

$$
\zeta(2 n+1)=\frac{(-1)^{n-1} 2^{2 n+1} \pi^{2 n}}{(2 n) !} \log \left(\prod_{k=1}^{n} S_{2 n+1}(k)^{a(2 n+1, k)}\right) .
$$

Example 1.2. We have

$$
\begin{aligned}
& \zeta(3)=4 \pi^{2} \log S_{3}(1) \\
& \zeta(5)=-\frac{4 \pi^{4}}{3} \log \left(S_{5}(1) S_{5}(2)^{11}\right) \\
& \zeta(7)=\frac{8 \pi^{6}}{45} \log \left(S_{7}(1) S_{7}(2)^{57} S_{7}(3)^{302}\right) .
\end{aligned}
$$

The above formula (1.1) was proved in $[\mathrm{KK}]$ previously.

Remark 1.3. By the formula

$$
S_{r}(k)=\prod_{l=0}^{k-1} S_{r-l}(1)^{\left(\begin{array}{c}
k-1 \\
l
\end{array}\right)(-1)^{l}}
$$

for $1 \leq k<r$ (cf. §2), we can also express $\zeta(2 n+1)$ in terms of $S_{l}(1)$ $(2 \leq l \leq 2 n+1)$ :

$$
\zeta(2 n+1)=\frac{(-1)^{n-1} 2^{2 n+1} \pi^{2 n}}{(2 n) !} \log \left(\prod_{l=2}^{2 n+1} S_{l}(1)^{b(2 n+1, l)}\right)
$$

with $b(2 n+1, l) \in \mathbf{Z}$.

Example 1.4. Since $S_{5}(2)=S_{5}(1) S_{4}(1)^{-1}$ (see §2),

$$
\zeta(5)=-\frac{4 \pi^{4}}{3} \log \left(S_{5}(1)^{12} S_{4}(1)^{-11}\right) .
$$

Next, let $\chi$ be a non-trivial primitive Dirichlet character modulo $N$, and

$$
L(s, \chi)=\sum_{n=1}^{\infty} \chi(n) n^{-s}
$$

the Dirichlet $L$-function. Then the values $L(r, \chi)$ for $r=1,2,3, \ldots$ are classified as

$$
L(r, \chi)=\left\{\begin{array}{lll}
\pi^{r} \cdot(\chi \text {-Bernoulli number }) & \cdots & \chi(-1)=(-1)^{r} \\
\text { "difficult" } & \cdots & \chi(-1)=(-1)^{r+1} .
\end{array}\right.
$$

Here "difficult" means that these values have not been calculated explicitly yet except for the $r=1$ case appearing in the Dirichlet's class number formula. 
We generalize Dirichlet's result to some difficult case.

THEOREM 1.5. Let $\chi$ be a primitive odd character modulo N. Then:

(1)

$$
L^{\prime}(-1, \chi)=-\frac{1}{2} \log \prod_{k=1}^{N-1}\left(S_{2}\left(\frac{k}{N}\right)^{N} S_{1}\left(\frac{k}{N}\right)^{k}\right)^{\chi(k)}
$$

(2)

$$
L(2, \chi)=\frac{2 \pi i \tau(\chi)}{N^{2}} \log \prod_{k=1}^{N-1}\left(S_{2}\left(\frac{k}{N}\right)^{N} S_{1}\left(\frac{k}{N}\right)^{k}\right)^{\bar{\chi}(k)}
$$

Example 1.6. We have

$$
\begin{aligned}
L\left(2,\left(\frac{-4}{*}\right)\right) & =-\frac{\pi}{4} \log \left(S_{2}\left(\frac{1}{4}\right)^{4} S_{1}\left(\frac{1}{4}\right) S_{2}\left(\frac{3}{4}\right)^{-4} S_{1}\left(\frac{3}{4}\right)^{-3}\right) \\
& =\frac{\pi}{4} \log \left(2^{3} S_{2}\left(\frac{1}{4}\right)^{-8}\right), \\
L\left(2,\left(\frac{-3}{*}\right)\right) & =-\frac{2 \sqrt{3} \pi}{9} \log \left(S_{2}\left(\frac{1}{3}\right)^{3} S_{1}\left(\frac{1}{3}\right) S_{2}\left(\frac{2}{3}\right)^{-3} S_{1}\left(\frac{2}{3}\right)^{-2}\right) \\
& =\frac{4 \sqrt{3} \pi}{9} \log \left(3 S_{2}\left(\frac{1}{3}\right)^{-3}\right),
\end{aligned}
$$

where we used the following relations (see §2):

$$
\begin{aligned}
S_{2}(1-x) & =S_{2}(1+x)^{-1} \\
& =\left(S_{2}(x) S_{1}(x)^{-1}\right)^{-1} \\
& =S_{2}(x)^{-1} S_{1}(x) .
\end{aligned}
$$
Then:

THEOREM 1.7. Let $\chi$ be a non-trivial primitive even character modulo $N$.

(1)

$$
L^{\prime}(-2, \chi)=-\frac{1}{2} \log \prod_{k=1}^{N-1}\left(S_{3}\left(\frac{k}{N}\right)^{2 N^{2}} S_{2}\left(\frac{k}{N}\right)^{2 N k-3 N^{2}} S_{1}\left(\frac{k}{N}\right)^{k^{2}}\right)^{\chi(k)} .
$$

(2)

$$
L(3, \chi)=\frac{2 \pi^{2} \tau(\chi)}{N^{3}} \log \prod_{k=1}^{N-1}\left(S_{3}\left(\frac{k}{N}\right)^{2 N^{2}} S_{2}\left(\frac{k}{N}\right)^{2 N k-3 N^{2}} S_{1}\left(\frac{k}{N}\right)^{k^{2}}\right)^{\bar{\chi}(k)}
$$


Example 1.8.

$$
\begin{aligned}
L\left(3,\left(\frac{12}{*}\right)\right)= & \frac{\sqrt{3} \pi^{2}}{432} \log \left(S_{3}\left(\frac{1}{12}\right)^{288} S_{2}\left(\frac{1}{12}\right)^{-408} S_{1}\left(\frac{1}{12}\right) S_{3}\left(\frac{5}{12}\right)^{-288}\right. \\
& \times S_{2}\left(\frac{5}{12}\right)^{312} S_{1}\left(\frac{5}{12}\right)^{-25} S_{3}\left(\frac{7}{12}\right)^{-288} S_{2}\left(\frac{7}{12}\right)^{264} S_{1}\left(\frac{7}{12}\right)^{-49} \\
& \left.\times S_{3}\left(\frac{11}{12}\right)^{288} S_{2}\left(\frac{11}{12}\right)^{-164} S_{1}\left(\frac{11}{12}\right)^{121}\right)
\end{aligned}
$$

Thus the values $S_{r}(a)$ for $a \in \mathbf{Q}$ satisfying $0<a<r$ are quite interesting in relation to zeta values. We formulate our expectation as

Expectation 1.9. $S_{r}(a) \in \overline{\mathbf{Q}}$ for $a \in \mathbf{Q}$ satisfying $0<a<r$.

The situation would become transparent when we generalize it as below: EXPECTATION 1.10.
$k_{i}=0,1, \ldots, N-1$.

It is easy to see that Expectation 1.9 is contained in Expectation 1.10 for $\underline{\omega}=(1, \ldots, 1)$, and Expectation 1.10 clearly indicates that we are studying division values of multiple sine functions.

We note that Shintani $[\mathrm{Sh}]$ deeply studied $S_{2}(x,(1, \varepsilon))$ for a fundamental unit $\varepsilon$ of a real quadratic field. In particular, he showed its appearance in the expression for a special value of a suitable $L$-function, and he obtained certain algebraicity such as

$$
S_{2}\left(\frac{1}{3},(1, \varepsilon)\right) S_{2}\left(1+\frac{\varepsilon}{3},(1, \varepsilon)\right) S_{2}\left(\frac{2+2 \varepsilon}{3},(1, \varepsilon)\right)=\sqrt{\frac{\frac{1+\sqrt{21}}{2}-\sqrt{\frac{3+\sqrt{21}}{2}}}{2}}
$$

for $\varepsilon=\frac{5+\sqrt{21}}{2}$, which is the fundamental unit of $\mathbf{Q}(\sqrt{21})$. Moreover, Shintani studied Kronecker's Jugendtraum for a real quadratic field by using $S_{2}(x,(1, \varepsilon))$ (he denoted it by $F(x ;(1, \varepsilon))^{-1}$ ). It might be valuable to report the following general product formula

$$
\prod_{\substack{k_{1}, \ldots, k_{r}=0 \\\left(k_{1}, \ldots, k_{r}\right) \neq(0, \ldots, 0)}}^{N-1} S_{r}\left(\frac{k_{1} \omega_{1}+\cdots+k_{r} \omega_{r}}{N} ;\left(\omega_{1}, \ldots, \omega_{r}\right)\right)=N
$$

for an integer $N \geq 2$. (See $\S 2$.) 
THEOREM 1.11. (1) Expectations 1.9 and 1.10 are valid for $r=1$.

(2) Expectations 1.9 and 1.10 are valid for $r=2$ with $N=2$. Actually

$$
S_{2}\left(\frac{\omega_{1}}{2} ; \underline{\omega}\right)=S_{2}\left(\frac{\omega_{2}}{2} ; \underline{\omega}\right)=\sqrt{2}
$$

and

$$
S_{2}\left(\frac{\omega_{1}+\omega_{2}}{2} ; \underline{\omega}\right)=1 .
$$

Remark 1.12. This paper was referred to in $[\mathrm{KK}]$ as a preprint in 2001 .

\section{Multiple sine functions}

The basic properties of multiple sine functions were proved in $[\mathrm{K}]$ and $[\mathrm{KK}]$. Here we recall some of them.

THEOREM 2.1 [KK, Theorem 2.1]. The multiple sine function $S_{r}(x, \underline{\omega})$ satisfies the following identities:

(a) For $\underline{\omega}=\left(\omega_{1}, \ldots, \omega_{r}\right) \in \mathbf{R}_{+}^{r}$ put $\underline{\omega}(i)=\left(\omega_{1}, \ldots, \omega_{i-1}, \omega_{i+1}, \ldots, \omega_{r}\right) \in \mathbf{R}_{+}^{r-1}$, then we have

$$
S_{r}\left(x+\omega_{i}, \underline{\omega}\right)=S_{r}(x, \underline{\omega}) S_{r-1}(x, \underline{\omega}(i))^{-1},
$$

where we put $S_{0}(x, \cdot) \equiv-1$.

(b) For a positive integer $N$, we have

$$
S_{r}(N x, \underline{\omega})=\prod_{0 \leq k_{i} \leq N-1} S_{r}\left(x+\frac{\mathbf{k} \cdot \underline{\omega}}{N}, \underline{\omega}\right),
$$

(c)

where the product is taken over the vectors $\mathbf{k}=\left(k_{1}, \ldots, k_{r}\right)$.

$$
\prod_{\substack{0 \leq k_{i} \leq N-1 \\ \mathbf{k} \neq \mathbf{0}}} S_{r}\left(\frac{\mathbf{k} \cdot \underline{\omega}}{N}, \underline{\omega}\right)=N
$$

(d)

$$
S_{r}(0, \underline{\omega})=0 .
$$

(e) We have for any $c>0$ the homogeneity

$$
S_{r}(c x, c \underline{\omega})=S_{r}(x, \underline{\omega}) .
$$

TheOREM 2.2. (a) For $r \geq 2$ we have

$$
S_{r}(x+1)=S_{r}(x) S_{r-1}(x)^{-1} .
$$


(b)

$$
S_{r}(2 x)=\prod_{k=0}^{r} S_{r}\left(x+\frac{k}{2}\right)^{\left(\begin{array}{l}
r \\
k
\end{array}\right)}
$$

(c) Put $Q_{r}(x)=(-1)^{r-1} \pi\left(\begin{array}{c}x-1 \\ r-1\end{array}\right)$, then

$$
\frac{S_{r}^{\prime}(x)}{S_{r}(x)}=Q_{r}(x) \cot (\pi x) \text {. }
$$

(d)

$$
S_{r}^{\prime \prime}(x)+\left(\pi Q_{r}(x)^{-1}-1\right) S_{r}^{\prime}(x)^{2} S_{r}(x)^{-1}-Q_{r}^{\prime}(x) Q_{r}(x)^{-1} S_{r}^{\prime}(x)+\pi Q_{r}(x) S_{r}(x)=0 .
$$

(e)

$$
\begin{aligned}
S_{2}(x) & =2 \pi x e^{-x} \prod_{n=1}^{\infty}\left(\left(1+\frac{x}{n}\right)^{n+1}\left(1-\frac{x}{n}\right)^{-n+1} e^{-2 x}\right) \\
& =2 \pi x e^{-x} \prod_{n=1}^{\infty}\left(\left(1+\frac{x}{n}\right)^{2 H_{n}}\left(1-\frac{x}{n}\right)^{2 H_{-n}} e^{-2 x}\right) .
\end{aligned}
$$

(f)

$$
\begin{aligned}
S_{3}(x) & =2 \pi e^{-\zeta^{\prime}(-2)} x e^{x^{2} / 4-(3 / 2) x} \prod_{n=1}^{\infty}\left(\left(1+\frac{x}{n}\right)^{n^{2} / 2+3 n / 2+1}\left(1-\frac{x}{n}\right)^{n^{2} / 2-3 n / 2+1} e^{x^{2} / 2-3 x}\right) \\
& =2 \pi e^{-\zeta^{\prime}(-2)} x e^{x^{2} / 4-(3 / 2) x} \prod_{n=1}^{\infty}\left(\left(1+\frac{x}{n}\right)^{3 H_{n}}\left(1-\frac{x}{n}\right)^{3 H_{-n}} e^{x^{2} / 2-3 x}\right) .
\end{aligned}
$$

Proof. The assertions (a) and (b) are immediate consequences from [KK, Theorem 2.1]. The differential equation (c) is proved in [KK, Theorem 2.15]. We compute from (c) that

$$
\begin{aligned}
\left(Q_{r}(x)^{-1} \frac{S_{r}^{\prime}}{S_{r}}(x)\right)^{\prime} & =(\cot \pi x)^{\prime} \\
& =-\frac{\pi}{\sin ^{2} \pi x} \\
& =-\pi\left(\cot ^{2}(\pi x)+1\right) \\
& =-\pi\left(\left(Q_{r}(x)^{-1} \frac{S_{r}^{\prime}}{S_{r}}(x)\right)^{2}+1\right),
\end{aligned}
$$

which gives the proof of (d). Finally (e) and (f) are deduced from [KK, Examples 3.6], where we express the normalized multiple sine functions $S_{r}(x)$ in 
terms of primitive multiple sine functions which are defined by the Hadamard product.

\section{The Riemann zeta function}

Lemma 3.1. There exist uniquely determined integers $a(r, k)$ such that

$$
x^{r-1}=\sum_{k=1}^{r-1} a(r, k)_{r} H_{x-k}
$$

with ${ }_{r} H_{x-k}=\frac{(x-k+r-1) \cdots(x-k+1)}{(r-1) !}$ for an indeterminate $x$. Indeed $a(r, k)$ are given as follows:

$$
a(r, k)=\sum_{l=1}^{k}(-1)^{k-l} l^{r-1}\left(\begin{array}{c}
r \\
k-l
\end{array}\right) .
$$

Moreover,

$$
a(r, r-k)=a(r, k) .
$$

Proof. The existence of $a(r, k)$ follows from the fact that the $(r-1)$ polynomials ${ }_{r} H_{x-k}(k=1, \ldots, r-1)$ are linearly independent over $\mathbf{Q}$. By putting $x=k$ in $(3.1)$, we have

$$
k^{r-1}=a(r, 1)\left(\begin{array}{c}
k+r-2 \\
r-1
\end{array}\right)+a(r, 2)\left(\begin{array}{c}
k+r-3 \\
r-1
\end{array}\right)+\cdots+a(r, k) \cdot 1 .
$$

This leads to

$$
a(r, k)=k^{r-1}-\sum_{j=1}^{k-1} a(r, j)\left(\begin{array}{c}
k+r-1-j \\
r-1
\end{array}\right) .
$$

Thus (3.2) is proved by induction on $k$. Next, from (3.1)

$$
(-x)^{r-1}=\sum_{k=1}^{r-1} a(r, k)_{r} H_{-x-k}
$$

and

$$
{ }_{r} H_{-x-k}=\frac{(-x-k+r-1) \cdots(-x-k+1)}{(r-1) !}=(-1)^{r-1}{ }_{r} H_{x-(r-k)},
$$

so 


$$
x^{r-1}=\sum_{k=1}^{r-1} a(r, k)_{r} H_{x-(r-k)}=\sum_{k=1}^{r-1} a(r, r-k)_{r} H_{x-k} .
$$

Hence, by the uniquenes of $a(r, k)$ we have $a(r, r-k)=a(r, k)$.

Example 3.2. For $x=n \in \mathbf{Z}$ and $r=2,3,4,5$ we have

$$
\begin{aligned}
n & ={ }_{2} H_{n-1}, \\
n^{2} & ={ }_{3} H_{n-1}+{ }_{3} H_{n-2}, \\
n^{3} & ={ }_{4} H_{n-1}+4{ }_{4} H_{n-2}+{ }_{4} H_{n-3}, \\
n^{4} & ={ }_{5} H_{n-1}+11_{5} H_{n-2}+11{ }_{5} H_{n-3}+{ }_{5} H_{n-4} .
\end{aligned}
$$

Proof of Theorem 1.1. For $r \geq 2$ we have by Lemma 3.1

$$
\begin{aligned}
\zeta(s+1-r) & =\sum_{n=1}^{\infty} \frac{n^{r-1}}{n^{s}} \\
& =\sum_{k=1}^{r-1} a(r, k) \sum_{n=1}^{\infty} \frac{{ }_{r} H_{n-k}}{n^{s}} \\
& =\sum_{k=1}^{r-1} a(r, k) \zeta_{r}(s, k),
\end{aligned}
$$

where $\zeta_{r}(s, k)$ is the multiple Hurwitz zeta function

$$
\zeta_{r}(s, k)=\sum_{n=0}^{\infty} \frac{{ }_{r} H_{n}}{(n+k)^{s}} .
$$

Thus we have

$$
\zeta^{\prime}(1-r)=\sum_{k=1}^{r-1} a(r, k) \log \Gamma_{r}(k) .
$$

In case $r=2 n+1$, it follows that

$$
\begin{aligned}
\zeta^{\prime}(-2 n) & =\sum_{k=1}^{2 n} a(2 n+1, k) \log \Gamma_{2 n+1}(k) \\
& =-\sum_{k=1}^{n} a(2 n+1, k) \log S_{2 n+1}(k) \\
& =-\log \left(\prod_{k=1}^{n} S_{2 n+1}(k)^{a(2 n+1, k)}\right)
\end{aligned}
$$


where we used $S_{2 n+1}(k)=\Gamma_{2 n+1}(k)^{-1} \Gamma_{2 n+1}(2 n+1-k)^{-1}$ and $a(2 n+1,2 n+$ $1-k)=a(2 n+1, k)$.

Example 3.3. We saw in $[\mathrm{KK}$, Theorem $3.8(\mathrm{c})]$ that

$$
\zeta(3)=4 \pi^{2} \log S_{3}(1)
$$

Combining this with the fact that

$$
S_{3}(1)=\sqrt{2} S_{3}\left(\frac{1}{2}\right)^{-4 / 3},
$$

which can be obtained by the facts

$$
\begin{aligned}
S_{3}(1) & =S_{3}\left(2 \cdot \frac{1}{2}\right) \\
& =S_{3}\left(\frac{1}{2}\right) S_{3}(1)^{3} S_{3}\left(\frac{3}{2}\right)^{3} S_{3}(2) \\
& =S_{3}(1)^{4} S_{3}\left(\frac{1}{2}\right)^{4} S_{2}\left(\frac{1}{2}\right)^{-3}
\end{aligned}
$$

and that $S_{2}\left(\frac{1}{2}\right)=\sqrt{2}$, we have

$$
\zeta(3)=\frac{16 \pi^{2}}{3} \log \left(S_{3}\left(\frac{1}{2}\right)^{-1} 2^{3 / 8}\right)
$$

which was proved in [KK, Theorem 3.8(b)] by another method (using a "primitive multiple sine function").

\section{Dirichlet $L$-functions for odd characters}

We prove the formula for $L(2, \chi)$ for odd characters. Since our method follows a proof for Dirichlet's result on $L(1, \chi)$ for even characters, we first recall it. We show the formula for $L^{\prime}(0, \chi)$. Then the result on $L(1, \chi)$ follows via the functional equation.

Let $\chi$ be a non-trivial primitive Dirichlet character modulo $N$. We have

$$
\begin{aligned}
L(s, \chi) & =\sum_{k=1}^{N-1} \chi(k) \sum_{m=0}^{\infty} \frac{1}{(m N+k)^{s}} \\
& =N^{-s} \sum_{k=1}^{N-1} \chi(k) \zeta\left(s, \frac{k}{N}\right),
\end{aligned}
$$


where

$$
\zeta(s, x)=\sum_{m=0}^{\infty} \frac{1}{(m+x)^{s}}
$$

is the Hurwitz zeta function. Hence

$$
L(0, \chi)=\sum_{k=1}^{N-1} \chi(k) \zeta\left(0, \frac{k}{N}\right)
$$

and

$$
\begin{aligned}
L^{\prime}(0, \chi) & =\sum_{k=1}^{N-1} \chi(k) \zeta^{\prime}\left(0, \frac{k}{N}\right)-(\log N) \sum_{k=1}^{N-1} \chi(k) \zeta\left(0, \frac{k}{N}\right) \\
& =\sum_{k=1}^{N-1} \chi(k) \zeta^{\prime}\left(0, \frac{k}{N}\right)-(\log N) L(0, \chi) .
\end{aligned}
$$

When $\chi$ is even, it holds that $L(0, \chi)=0$ (this is the reason of "difficult"), so we have

$$
\begin{aligned}
L^{\prime}(0, \chi) & =\sum_{k=1}^{N-1} \chi(k) \zeta^{\prime}\left(0, \frac{k}{N}\right) \\
& =\sum_{k=1}^{N-1} \chi(k) \log \Gamma_{1}\left(\frac{k}{N}\right) \\
& =\frac{1}{2} \sum_{k=1}^{N-1} \chi(k)\left(\log \Gamma_{1}\left(\frac{k}{N}\right)+\log \Gamma_{1}\left(\frac{N-k}{N}\right)\right) \\
& =-\frac{1}{2} \sum_{k=1}^{N-1} \chi(k) \log S_{1}\left(\frac{k}{N}\right) .
\end{aligned}
$$

This gives the Dirichlet's result.

Proof of Theorem 1.5. We prove (1), then (2) is obtained via the functional equation. Since

$$
\begin{aligned}
\zeta(s-1, x) & =\sum_{n=0}^{\infty} \frac{n+x}{(n+x)^{s}}=\sum_{n=0}^{\infty} \frac{n+1}{(n+x)^{s}}+(x-1) \sum_{n=0}^{\infty} \frac{1}{(n+x)^{s}} \\
& =\zeta_{2}(s, x)+(x-1) \zeta_{1}(s, x),
\end{aligned}
$$

we have

$$
\zeta^{\prime}(-1, x)=\zeta_{2}^{\prime}(0, x)+(x-1) \zeta_{1}^{\prime}(0, x)
$$


as $\zeta_{1}(s, x)=\zeta(s, x)$. Now that $\chi$ is odd and that $L(-1, \chi)=0$, we compute

$$
\begin{aligned}
& L^{\prime}(-1, \chi)=N \sum_{k=1}^{N-1} \chi(k) \zeta^{\prime}\left(-1, \frac{k}{N}\right) \\
& =N \sum_{k=1}^{N-1} \chi(k) \zeta_{2}^{\prime}\left(0, \frac{k}{N}\right)+N \sum_{k=1}^{N-1} \chi(k)\left(\frac{k}{N}-1\right) \zeta_{1}^{\prime}\left(0, \frac{k}{N}\right) \\
& =N \sum_{k=1}^{N-1} \chi(k) \log \Gamma_{2}\left(\frac{k}{N}\right)+N \sum_{k=1}^{N-1} \chi(k)\left(\frac{k}{N}-1\right) \log \Gamma_{1}^{\prime}\left(\frac{k}{N}\right) \\
& =N \sum_{k=1}^{N-1} \chi(k) \log \left(\Gamma_{2}\left(\frac{k}{N}\right) \Gamma_{1}\left(\frac{k}{N}\right)^{k / N-1}\right) \\
& =\frac{N}{2} \sum_{k=1}^{N-1} \chi(k) \log \left(\frac{\Gamma_{2}\left(\frac{k}{N}\right)}{\Gamma_{2}\left(1-\frac{k}{N}\right)} \frac{\Gamma_{1}\left(\frac{k}{N}\right)^{k / N-1}}{\Gamma_{1}\left(1-\frac{k}{N}\right)^{-k / N}}\right) \\
& =\frac{N}{2} \sum_{k=1}^{N-1} \chi(k) \log \left(\frac{\Gamma_{2}\left(\frac{k}{N}\right)}{\Gamma_{2}\left(2-\frac{k}{N}\right)}\left(\Gamma_{1}\left(\frac{k}{N}\right) \Gamma_{1}\left(1-\frac{k}{N}\right)\right)^{k / N-1}\right) \\
& =-\frac{N}{2} \sum_{k=1}^{N-1} \chi(k) \log \left(S_{2}\left(\frac{k}{N}\right) S_{1}\left(\frac{k}{N}\right)^{k / N-1}\right) \\
& =-\frac{N}{2} \sum_{k=1}^{N-1} \chi(k) \log \left(S_{2}\left(\frac{k}{N}\right) S_{1}\left(\frac{k}{N}\right)^{k / N}\right) \text {, }
\end{aligned}
$$

where we used the fact $S_{1}\left(\frac{k}{N}\right)=S_{1}\left(\frac{N-k}{N}\right)$ with $\chi(N-k)=-\chi(k)$.

\section{Dirichlet $L$-functions for even characters}

Proof of Theorem 1.7. We again show (1), then (2) is obtained via the functional equation. Since

$$
(n+x)^{2}=2{ }_{3} H_{n}+(2 x-3){ }_{2} H_{n}+(x-1)^{2}{ }_{1} H_{n},
$$

we have

$$
\zeta(s-2, x)=\sum_{n=0}^{\infty} \frac{(n+x)^{2}}{(n+x)^{s}}=2 \zeta_{3}(s, x)+(2 x-3) \zeta_{2}(s, x)+(x-1)^{2} \zeta_{1}(s, x) .
$$


Therefore we have

$$
\zeta^{\prime}(-2, x)=2 \zeta_{3}^{\prime}(0, x)+(2 x-3) \zeta_{2}^{\prime}(0, x)+(x-1)^{2} \zeta_{1}^{\prime}(0, x) .
$$

Now that $\chi$ is even and that $L(-2, \chi)=0$, we compute

$$
\begin{aligned}
L^{\prime}(-2, \chi) & =N^{2} \sum_{k=1}^{N-1} \chi(k) \zeta^{\prime}\left(-2, \frac{k}{N}\right) \\
& =N^{2} \sum_{k=1}^{N-1} \chi(k)\left(2 \zeta_{3}^{\prime}\left(0, \frac{k}{N}\right)+\left(2 \frac{k}{N}-3\right) \zeta_{2}^{\prime}\left(0, \frac{k}{N}\right)+\left(\frac{k}{N}-1\right)^{2} \zeta_{1}^{\prime}\left(0, \frac{k}{N}\right)\right) \\
& =N^{2} \sum_{k=1}^{N-1} \chi(k) \log \left(\Gamma_{3}\left(\frac{k}{N}\right)^{2} \Gamma_{2}\left(\frac{k}{N}\right)^{2 k / N-3} \Gamma_{1}\left(\frac{k}{N}\right)^{(k / N-1)^{2}}\right) \\
& =-\frac{1}{2} \log \prod_{k=1}^{N-1}\left(S_{3}\left(\frac{k}{N}\right)^{2 N^{2}} S_{2}\left(\frac{k}{N}\right)^{2 N k-3 N^{2}} S_{1}\left(\frac{k}{N}\right)^{k^{2}}\right)^{\chi(k)}
\end{aligned}
$$

\section{Division values of normalized multiple sines}

Proof of Theorem 1.11. Since $S_{1}(x, \omega)=2 \sin \left(\frac{\pi x}{\omega}\right)$ by $[\mathrm{KK}, \S 2]$, we have

$$
S_{1}\left(\frac{k \omega}{N}, \omega\right)=2 \sin \left(\frac{k \pi}{N}\right)=-i\left(e^{i \pi k / N}-e^{-i \pi k / N}\right) \in \overline{\mathbf{Q}},
$$

which leads to (1).

Recall that

$$
S_{2}\left(x,\left(\omega_{1}, \omega_{2}\right)\right)=\frac{\Gamma_{2}\left(\omega_{1}+\omega_{2}-x,\left(\omega_{1}, \omega_{2}\right)\right)}{\Gamma_{2}\left(x,\left(\omega_{1}, \omega_{2}\right)\right)} .
$$

First

$$
S_{2}\left(\frac{\omega_{1}+\omega_{2}}{2},\left(\omega_{1}, \omega_{2}\right)\right)=\frac{\Gamma_{2}\left(\frac{\omega_{1}+\omega_{2}}{2},\left(\omega_{1}, \omega_{2}\right)\right)}{\Gamma_{2}\left(\frac{\omega_{1}+\omega_{2}}{2},\left(\omega_{1}, \omega_{2}\right)\right)}=1
$$

Secondly

$$
S_{2}\left(\frac{\omega_{1}}{2},\left(\omega_{1}, \omega_{2}\right)\right)=\frac{\Gamma_{2}\left(\frac{\omega_{1}}{2}+\omega_{2},\left(\omega_{1}, \omega_{2}\right)\right)}{\Gamma_{2}\left(\frac{\omega_{1}}{2},\left(\omega_{1}, \omega_{2}\right)\right)}
$$


Here we use $([\mathrm{KK}, \S 2])$

$$
\Gamma_{2}\left(x+\omega_{2},\left(\omega_{1}, \omega_{2}\right)\right)=\Gamma_{2}\left(x,\left(\omega_{1}, \omega_{2}\right)\right) \Gamma_{1}\left(x, \omega_{1}\right)^{-1} .
$$

Then

$$
\Gamma_{2}\left(\frac{\omega_{1}}{2}+\omega_{2},\left(\omega_{1}, \omega_{2}\right)\right)=\Gamma_{2}\left(\frac{\omega_{1}}{2},\left(\omega_{1}, \omega_{2}\right)\right) \Gamma_{1}\left(\frac{\omega_{1}}{2}, \omega_{1}\right)^{-1}
$$

Hence

$$
S_{2}\left(\frac{\omega_{1}}{2},\left(\omega_{1}, \omega_{2}\right)\right)=\Gamma_{1}\left(\frac{\omega_{1}}{2}, \omega_{1}\right)^{-1}
$$

Now $([K K, \S 2])$

$$
\Gamma_{1}(x, \omega)=\frac{\Gamma\left(\frac{x}{\omega}\right)}{\sqrt{2 \pi}} \omega^{x / \omega-1 / 2}
$$

so

$$
\Gamma_{1}\left(\frac{\omega_{1}}{2}, \omega_{1}\right)=\frac{\Gamma\left(\frac{1}{2}\right)}{\sqrt{2 \pi}}=\frac{1}{\sqrt{2}} .
$$

Thus

$$
S_{2}\left(\frac{\omega_{1}}{2},\left(\omega_{1}, \omega_{2}\right)\right)=\sqrt{2}
$$

Remark 6.1. A suitable restriction on the form of division points such as made in Expectation 1.10 will be needed as the following example shows:

$$
S_{2}(2,(1, \sqrt{2})) \notin \overline{\mathbf{Q}} \text {. }
$$

By this example, we must seriously look at $S_{r}\left(a_{1} \omega_{1}+\cdots+a_{r} \omega_{r} ;\left(\omega_{1}, \ldots, \omega_{r}\right)\right)$ for general $a_{i} \in \mathbf{Q}$. The proof of $(6.1)$ is given by

$$
\frac{S_{2}(2,(1, \sqrt{2}))}{S_{2}(1,(1, \sqrt{2}))}=\frac{S_{2}(1+1,(1, \sqrt{2}))}{S_{2}(1,(1, \sqrt{2}))}=S_{1}(1, \sqrt{2})^{-1}=\left(2 \sin \frac{\pi}{\sqrt{2}}\right)^{-1} \notin \overline{\mathbf{Q}},
$$

where

$$
\begin{aligned}
2 \sin \frac{\pi}{\sqrt{2}} & =-i\left(e^{i \pi / \sqrt{2}}-e^{-i \pi / \sqrt{2}}\right) \\
& =-i\left((-1)^{1 / \sqrt{2}}-\left((-1)^{1 / \sqrt{2}}\right)^{-1}\right)
\end{aligned}
$$

and we used the transcendency result of Gelfond-Schneider $(-1)^{1 / \sqrt{2}} \notin \overline{\mathbf{Q}}$. Moreover we appeal to the following facts: 


$$
\begin{aligned}
& S_{2}\left(\omega_{1},\left(\omega_{1}, \omega_{2}\right)\right)=\sqrt{\frac{\omega_{2}}{\omega_{1}}}, \\
& S_{2}\left(\omega_{2},\left(\omega_{1}, \omega_{2}\right)\right)=\sqrt{\frac{\omega_{1}}{\omega_{2}}} .
\end{aligned}
$$

In particular

$$
S_{2}(1,(1, \sqrt{2}))=2^{1 / 4} \in \overline{\mathbf{Q}}
$$

Thus we obtain (6.1).

\title{
REFERENCES
}

[B] E. W. BARnes, On the theory of the multiple gamma function, Trans. Cambridge Philos. Soc. 19 (1904), 374-425.

[K] N. Kurokawa, Gamma factors and Plancherel measures, Proc. Japan Acad., Ser. A. 68 (1992), 256-260.

[KK] N. Kurokawa and S. Koyama, Multiple sine functions, Forum Math. 15 (2003), 839876.

[KW] N. Kurokawa and M. Wakayama, On $\zeta(3)$, J. Ramanujan Math. Soc. 16 (2001), 205214.

[M] YU. I. Manin, Lectures on zeta functions and motives (according to Deninger and Kurokawa), Asterisque 228 (1995), 121-163.

[Sh] T. Shintani, On a Kronecker limit formula for real quadratic fields, J. Fac. Sci. Univ. Tokyo, 24 (1977), 167-199.

\author{
2-5-27 Hayabuchi, Tsuzuki-Ku \\ YOKOHAMA 224-0025 \\ JAPAN \\ E-mail: koyama@tmtv.ne.jp \\ Department of Mathematics \\ Tokyo Institute of Technology \\ OH-OKayama, Meguro-Ku \\ TOKYO 152-8551 \\ JAPAN \\ E-mail: kurokawa@math.titech.ac.jp
}

